\title{
Diversity of haemoprotozoan parasites infecting the wildlife of South Africa
}

\author{
D. James Harris ${ }^{1,2}$, Ali Halajian ${ }^{3}$, Joana L. Santos ${ }^{1}$, Lourens H. Swanepoel ${ }^{4}$, Peter John Taylor S6,7 $^{5,7}$ \\ and Raquel Xavier ${ }^{1}$
}

\author{
${ }^{1}$ CIBIO/InBIO, Centro de Investigação em Biodiversidade e Recursos Genéticos, Campus Agrário de Vairão, Vairão, Portugal; \\ ${ }^{2}$ Departamento de Biologia, Faculdade de Ciências da Universidade do Porto, Porto, Portugal; \\ ${ }^{3}$ Department of Biodiversity, University of Limpopo, South Africa; \\ ${ }^{4}$ Department of Zoology, School of Mathematical and Natural Sciences, University of Venda, Thohoyandou, South Africa; \\ ${ }^{5}$ NRF/DET SARCHI Chair on Biodiversity Value and Change, School of Mathematical and Natural Sciences, University of Venda, \\ Thohoyandou, South Africa; \\ ${ }^{6}$ Core Team Member, Centre for Invasion Biology, Stellenbosch University, Stellenbosch, Republic of South Africa; \\ ${ }^{7}$ Honorary Research Associate, University of KwaZulu Natal, Durban, Republic of South Africa
}

\begin{abstract}
Tissue samples from wildlife from South Africa were opportunistically collected and screened for haemoprotozoan parasites using nonspecific PCR primers. Samples of 127 individuals were tested, comprising over 50 different species. Haemogregarines were the most commonly identified parasites, but sarcocystids and piroplasmids were also detected. Phylogenetic analyses estimated from the 18S rDNA marker highlighted the occurrence of several novel parasite forms and the detection of parasites in novel hosts. Phylogenetic relationships, which have been recently reviewed, appear to be much more complex than previously considered. Our study highlights the high diversity of parasites circulating in wildlife in this biodiverse region, and the need for further studies to resolve taxonomic issues.
\end{abstract}

Keywords: Babesia, Theileria, Hepatozoon, Sarcocystis, Cytauxzoon, phylogeny, 18S rDNA

South Africa is the third most biologically diverse country in the world for terrestrial species. It includes three of the 35 recognised biodiversity hotspots, the Cape Floristic region, the Succulent Karoo and the Maputoland-Floristic region (Mittermeier et al. 2004). Yet despite this wellknown biodiversity richness, parasite diversity remains poorly known. One of the few known patterns of parasite diversity is a link with overall biodiversity, leading to the 'host diversity' begets parasite diversity" principle, and indicating that parasite diversity should also be very high in the region.

Parasites have dual interests for conservation biologists. Firstly parasite-driven declines in wildlife have become increasingly common (Pedersen and Fenton 2007), illustrated by multiple pathogens being implicated in the global decline of amphibian populations (e.g. Hoverman et al. 2012). Second, parasites also represent a major component of biodiversity. Because of their obligate relationships with their hosts, parasites are particularly at risk through co-extinctions, where the loss of a species leads to the loss of dependent species so that a cascading extinction effect occurs.
Models suggest that co-extinction may be the most common form of biodiversity loss (Dunn et al. 2009), yet paradoxically lack of basic knowledge of most parasite groups prevents risk assessments for example - Dobson et al. (2008) state "We have no credible way of estimating how many parasitic protozoa ... exist". Part of the problem in estimating parasite diversity has classically been that, especially for endoparasites, often only some life stages can be identified in host blood or tissue samples, making identification based on morphological characters difficult. Molecular tools, particularly DNA sequencing approaches, should help overcome these problems, but molecular studies have lagged behind those of free-ranging organisms (Criscione et al. 2005).

Currently five groups of haemoprotozoan parasites are recognised, one group of euglenozoan flagellates and four groups of apicomplexan parasites (reviewed in O'Donoghue 2017). These infect all terrestrial vertebrate groups and use a variety of invertebrates including Diptera, ticks and leeches as vectors. Despite their medical and veterinary importance, only a few studies have screened verte- 
Table 1. List of positive samples, host identification and locality

\begin{tabular}{|c|c|c|c|}
\hline Code & Species Tissue & Tissue & Locality \\
\hline \multicolumn{4}{|l|}{ Mammals } \\
\hline Bush915 & Aepyceros melampus (Lichtenstein) & $\mathbf{M} / \mathrm{L}$ & Pullen Farm, Nelspruit, Mpumalanga province \\
\hline RLMar152 & Aethomys chrysophilus (de Winton) & $\mathbf{M} / \mathrm{L}$ & Louis Trichardt, Limpopo province \\
\hline RLMar162 & Aethomys chrysophilus (de Winton) & $\mathbf{M} / \mathbf{L}$ & Louis Trichardt, Limpopo province \\
\hline RVenJa151 & Aethomys chrysophilus (de Winton) & $\mathbf{M} / \mathbf{L}$ & Vyeboom village, Limpopo province \\
\hline RLMar1517 & Aethomys ineptus Thomas et Wroughton & $\mathbf{M} / \mathbf{L}$ & Louis Trichardt, Limpopo province \\
\hline MonN1Oc14 & Ichneumia albicauda (Cuvier) & $\mathbf{M} / \mathbf{L}$ & N1, Close to Louis Trichardt, Limpopo province \\
\hline Mon1015 & Ichneumia albicauda (Cuvier) & $\mathbf{M} / \mathrm{L}$ & Groothoek, Dendron, R521, Limpopo province \\
\hline RKR37416 & Ichneumia albicauda (Cuvier) & M & R37, Limpopo province \\
\hline BatL152 & Neoromicia zuluensis (Roberts) & $\mathbf{M} / \mathbf{L}$ & Louis Trichardt, Limpopo province \\
\hline SqVenF15 & Paraxerus cepapi (Smith) & $\mathbf{M} / \mathbf{L}$ & Vyeboom village, Limpopo province \\
\hline RhaTF142 & Rhabdomys sp. & $\mathbf{M} / \mathbf{L}$ & Tweefontein, Polokwane, Limpopo province \\
\hline RLMar155 & Unidentified rodent & $\mathbf{M} / \mathbf{L}$ & Louis Trichardt, Limpopo province \\
\hline GSqR34D14 & Xerus inauris (Zimmermann) & $\mathbf{M} / \mathbf{L}$ & R34, Wesselsbron, Free State province \\
\hline \multicolumn{4}{|l|}{ Reptiles } \\
\hline Sn-Najann4 & Naja annulifera Peters & $\mathbf{M} / \mathbf{L}$ & Louis Trichardt, Limpopo province \\
\hline Sn-CobO14 & Naja mossambica Peters & $\mathbf{M} / \mathbf{L}$ & Claudius Hoop 106L, Limpopo province \\
\hline Terr316 & Pelomedusa subrufa (Lacépède) & $\mathbf{M} / \mathbf{L}$ & R71, Onverwacht junction, Limpopo province \\
\hline Terr516 & Pelusios sinuatus (Smith) & $\mathbf{M} / \mathrm{L}$ & Flag Boshielo dam, Schuinsdrai nature reserve, Limpopo province \\
\hline
\end{tabular}

$\mathrm{M}$ - muscle tissue; $\mathrm{L}$ - liver. Tissues shown in bold were positive for the respective hosts.

brates from southern Africa for these parasites (e.g. Bosman et al. 2010, Van As et al., 2013, Harris et al. 2017).

Most other assessments of apicomplexan parasites in southern Africa have targeted specific species and parasites (e.g. Dubey et al. 2014, Netherlands et al. 2014, Cook et al. 2016), which means that presence of parasites in many species remains either completely unknown or is based only on older microscopic screening surveys (e.g. Basson et al. 1967). This motivated the current study, in which we screened various vertebrate species for infection by these parasites, using primers that are known to amplify across the different groups (e.g. Harris et al. 2013) and from different sources including muscle and blood (Maia et al. 2014).

\section{MATERIALS AND METHODS}

Sampling was opportunistic, consisting of either samples collected as road kills, or from tissue banks from various collections (Table 1). A few of the road-killed specimens could not be identified to the species level due to the poor state of the sample, but were still tested. When available, both liver and muscle tissues were sampled from the same individual. The molecular approach followed standard procedures used in other screening studies (e.g. Harris et al. 2012). DNA was extracted from the different tissues using high salt procedures, which have been shown to be as effective as more expensive kits for extracting parasite DNA from blood and muscle tissues (Maia et al. 2014).

Polymerase Chain Reaction (PCR) amplification was performed using the primers HepF300 and HepR900 (Ujvari et al. $2004)$ with 35 cycles consisting of $94^{\circ} \mathrm{C}(30 \mathrm{~s}), 60^{\circ} \mathrm{C}(30 \mathrm{~s})$ and $72^{\circ} \mathrm{C}(1 \mathrm{~min})$. These primers were chosen as they have been demonstrated to amplify a wide taxonomic range of apicomplexan parasites from reptilian, amphibian and mammalian hosts (e.g. Harris et al. 2013, Maia et al. 2014). Negative and positive controls were run with each reaction and products sequenced by a commercial company (Genewiz, Takeley, UK). Electropherograms were checked by eye and compared against published sequences on GenBank using BLAST. All new sequences were submitted to GenBank numbers MH924604 - MH924623.

Since various diverse parasites were detected, separate phylogenetic analyses were performed for each of the parasite groups recovered. For each data set new sequences produced herein were aligned with related sequences from GenBank, identified through the BLAST search. Sequences were aligned using the ClustalW software implemented in Geneious 4.8.5 (Biomatters Ld.). This led to the production of three data sets, containing 110, 28 and 38 taxa, which were 577,618 and 625 base pairs long, respectively.

Phylogenetic relationships were estimated using maximum likelihood (ML) and Bayesian inference. We implemented the models of sequence evolution selected by PartitionFinder 1.1.1 (Lanfear et al. 2012). ML analyses were performed with RAxML v 7.4.2 (Stamatakis 2006) with 10 random addition replicates, and node support estimated with 1,000 nonparametric bootstrap replicates. Bayesian inference was implemented using MrBayes v3.2 (Huelsenbeck and Ronquist 2001), and run for 1 million generations. After $25 \%$ burn in, remaining trees were combined in a $50 \%$ majority rule consensus.

\section{RESULTS}

Of the 236 tissue samples analysed, from 127 individuals, 20 produced amplified PCR products that led to identifiable parasite sequences. Haemogregarines were the most commonly identified parasites (Fig. 1). In reptiles, four hosts, two freshwater turtles Pelomedusa subrufa (Lacépède) and Pelusios sinuatus (Smith), and two snakes (Naja annulifera Peters and Naja mossambica Peters) were infected with haemoprotozoans. The haplotypes recovered from the freshwater turtles were sister taxa and strongly supported as a member of the monophyletic Haemogregarinidae clade that has been identified in previous molecular assessments (e.g. Karadjian et al. 2015).

Taxonomy within haemogregarines is complex (see discussion) but remaining haemogregarines were assigned to a paraphyletic Hepatozoon Miller, 1908 (see Maia et al. 


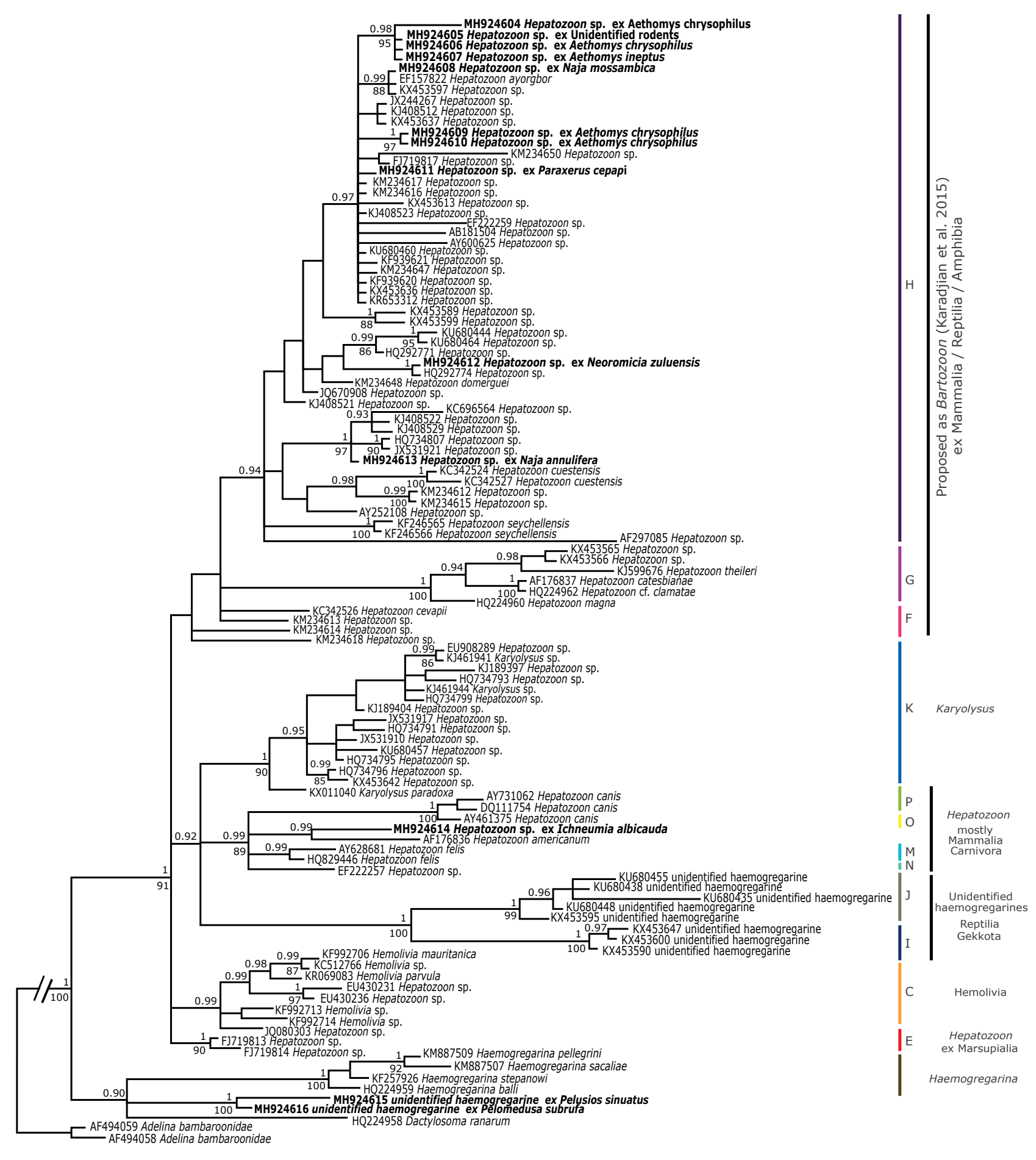

0.02

Fig. 1. Estimate of relationships of haemogregarines derived from partial 18S rRNA gene sequences using a Bayesian approach. Values above nodes correspond to bootstrap values from an ML approach, and those below nodes correspond to posterior probabilities. New sequences generated for this study are shown in bold. Right-hand side lettres correspond to the major groups identified by Maia et al. (2016).

2016). In N. mossambica the parasitic lineage found was most closely related to Hepatozoon ayorgbor Sloboda, Kamler, Bulantová, Votýpka et Modrý, 2007 described from a snake in Ghana (Sloboda et al. 2007). The lineage found infecting $N$. annulifera was closely related to other
Hepatozoon spp. recovered from various snakes and lizards from North Africa (Maia et al. 2011). A white-tailed mongoose Ichneumia albicauda (Cuvier) was infected by a species clearly sister to Hepatozoon felis Patton, 1908, known from a wide range of felids. One sample of the Zulu 


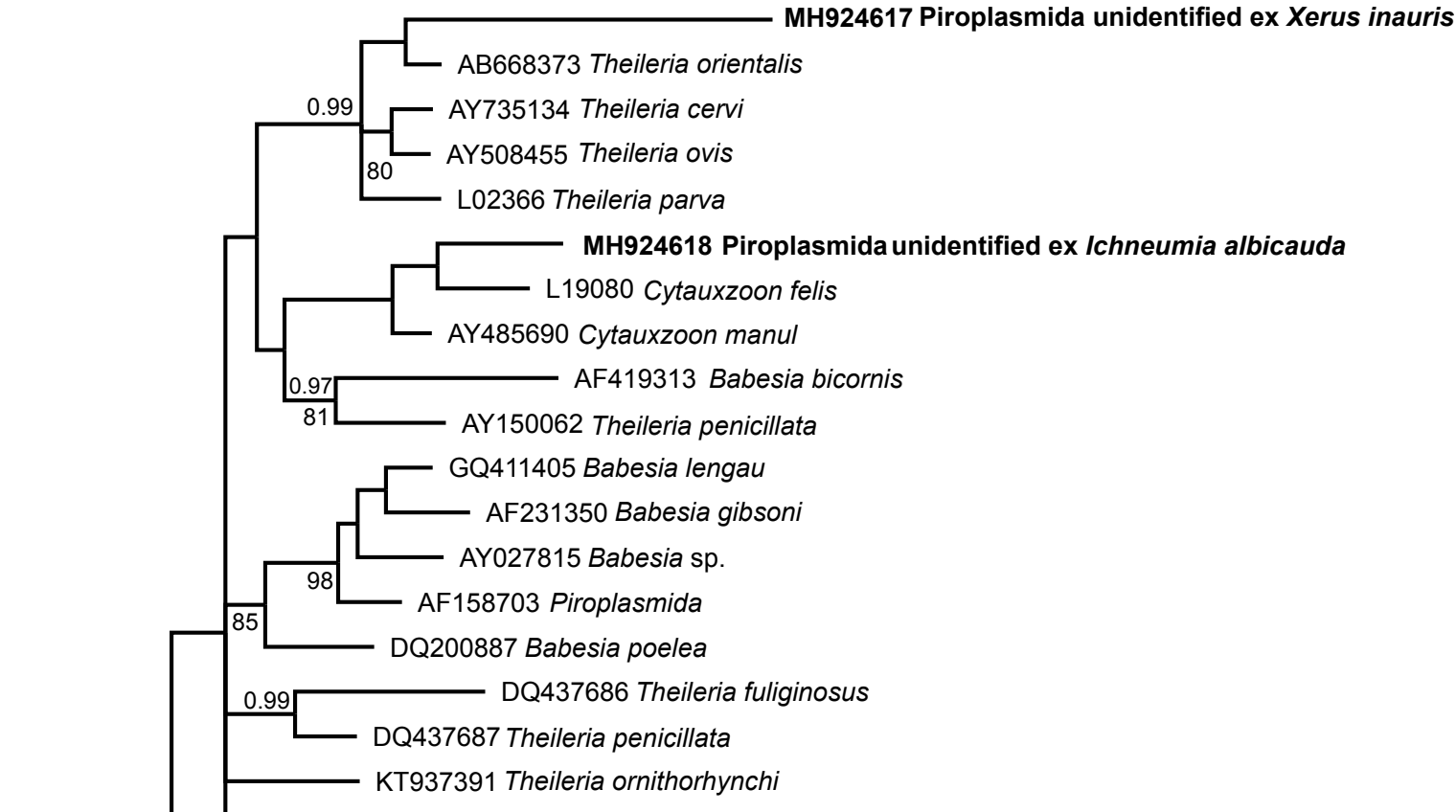

MH924619 Piroplasmida unidentified ex Rhabdomys sp.

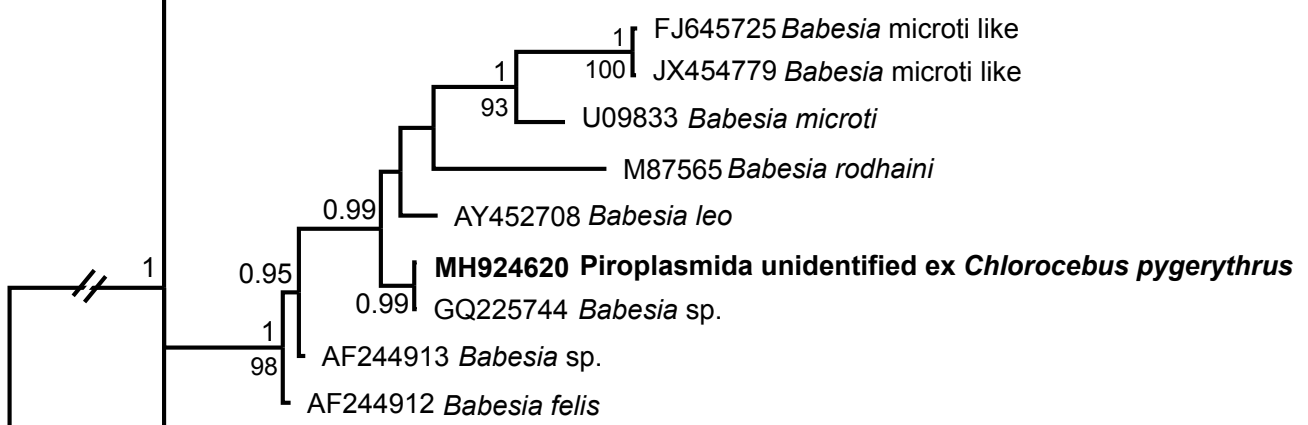

DQ402155 Babesia bennetti

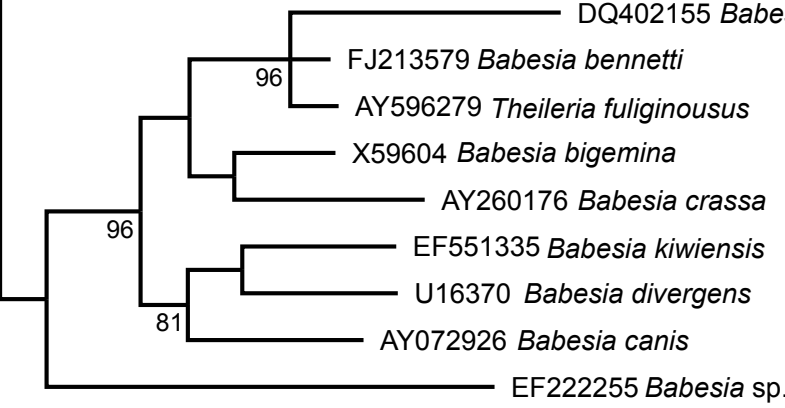

Plasmodium ovale

0.07

Fig. 2. Estimate of relationships of piroplasmids derived from partial 18S rRNA gene sequences using a Bayesian approach. Values above nodes correspond to bootstrap values from an ML approach, and those below nodes correspond to posterior probabilities. New sequences generated for this study are shown in bold.

pipistrelle bat, Neoromicia zuluensis (Roberts), gave a positive result, with a lineage similar to one obtained from a snake from the Seychelles. All other positive samples were from rodents and fell within a clade predominantly consisting of isolates of Hepatozoon identified from other rodents and snakes.

Four different piroplasmids were identified, belonging to the families Babesiidae and Theileridae (Fig. 2). Relationships of accepted genera within these families are complex, with six broad clades identified (reviewed in O'Donoghue 2017). One of these consists of species of Theileria Bettencourt, França et Borges, 1907 or Cytauxzoon Kier, 1979 from carnivores, and one sample from the white-tailed mongoose fell within this group, sister to Cytauxzoon felis Kier, 1979. Another sample from a vervet monkey, Chlorocebus pygerythrus (Cuvier), was identical to a sample of Babesia sp. from an olive baboon Papio anubis (Lesson). Two other samples from a grass mouse, 


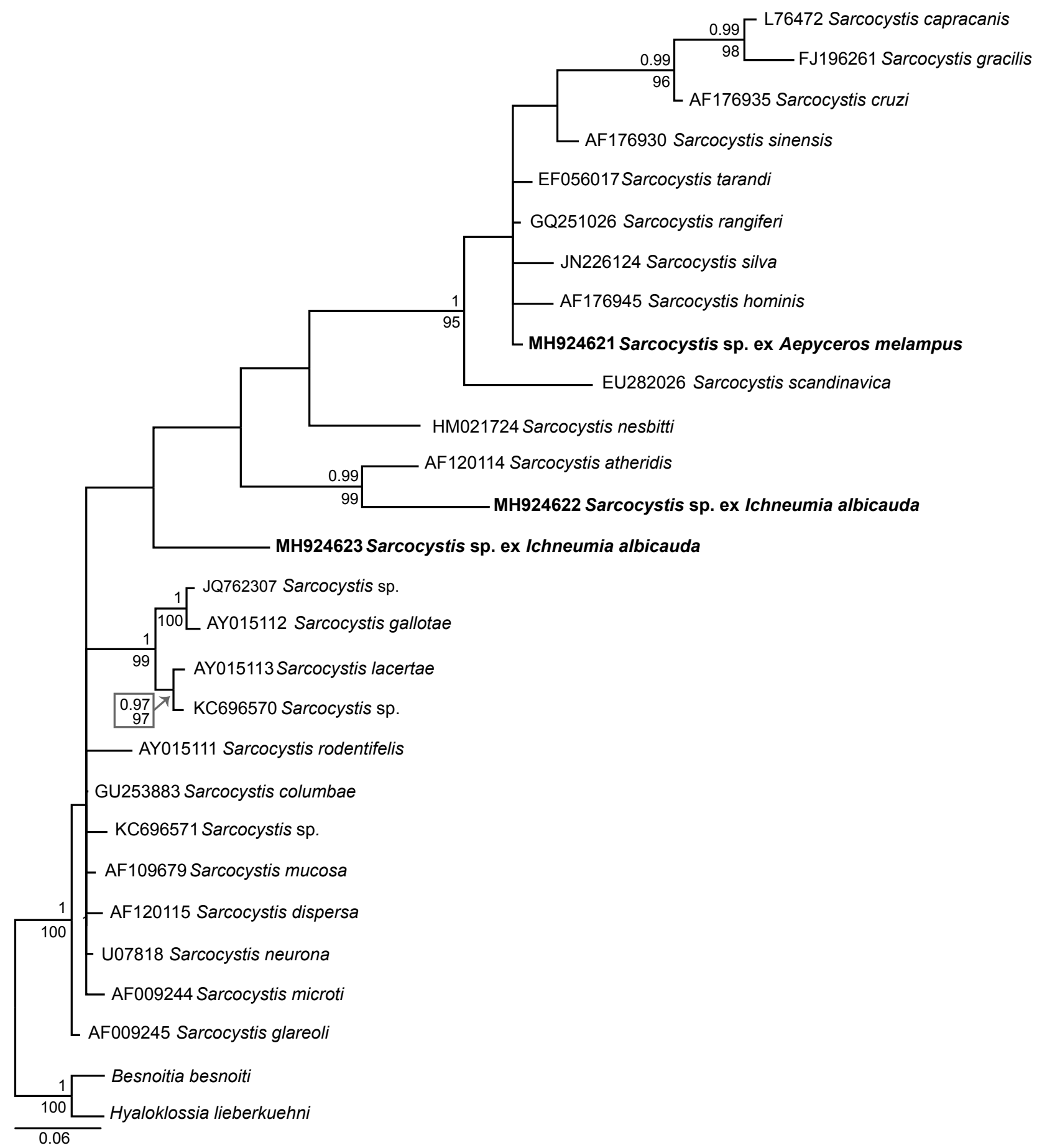

Fig. 3. Estimate of relationships of sarcocystids derived from partial 18S rRNA gene sequences using a Bayesian approach. Values above nodes correspond to bootstrap values from an ML approach, and those below nodes correspond to posterior probabilities. New sequences generated for this study are shown in bold.

Rhabdomys sp., and the Cape ground squirrel, Xerus inauris (Zimmermann), were infected with parasites that were distinct from published sequences but related to two different lineages of Theileria (Fig. 2).

The remaining positive samples were all phylogenetically related to the Sarcocystidae. One sample from the impala Aepyceros melampus (Lichtenstein) was identical to Sarcocystis truncata Gjerde, 2013 (originally reported as $S$. rangiferi) from red deer, Cervus elaphus Linnaeus. Two white-tailed mongoose were infected, each by a species from very different parasite lineage. One lineage was sister taxon to Sarcocystis atheridis Šlapeta, Modrý et Koudela, 1999 from Nitsche's bush viper, Atheris nitschei Tornier, whereas the relationships of the other lineage are not clear (Fig. 3).

\section{DISCUSSION}

The primers used in this assessment allowed the detection of multiple and phylogenetically diverse lineages of the Apicomplexa. Haemogregarines are composed of 
the genera Haemogregarina Danilewsky, 1885, Desseria Siddall, 1995 and Cyrilia Lainson, 1981 (Haemogregarinidae), Hepatozoon (Hepatozoidae), Hemolivia Petit, Landau, Baccam et Lainson, 1990 and Karyolysus Labbé, 1894 (Karyolysidae), and Dactylosoma Labbé, 1894 and Babesiosoma Jakowska et Nigrelli, 1956 (Dactylomatidae) (see Smith and Desser 1997, Telford 2009). Parasites of the genus Haemogregarina are known to use chelonians as the vertebrate host. In this study, however, both samples from freshwater terrapins, Pelusios sinuatus and Pelomedusa subrufa, appear to be infected with parasites that formed a distinct lineage that was more closely related to Dactylosoma than to different species of Haemogregarina. Further studies on South African terrapins using both microscopy and molecular tools are clearly needed to better identify these parasites.

While the taxonomy within the Haemogregarinidae is not controversial, the situation in the remaining families is far from stable. The genus Hepatozoon is the most widely reported and the most speciose, with over 300 species (Telford 2009). In a recent review of phylogenetic relationships, O’Donoghue (2017) reported that Hepatozoon was paraphyletic, with one group using carnivores as the vertebrate host related to Karyolysus, and another using non-carnivores related to Hemolivia. Karadjian et al. (2015) had previously proposed that the lineage found in carnivores should remain Hepatozoon, and the other lineage of parasites considered Hepatozoon should be placed in a new genus Bartozoon Karadjian, Chavatte et Landau, 2015. However, the situation is far more complex, since relationships between Hepatozoon and Hemolivia are not stable, and tend to change depending on which outgroup is used (as highlighted in Maia et al. 2016), and an entire new lineage that use geckos as vertebrate hosts has recently been identified (Tomé et al. 2016), which does not fit into the newly proposed taxonomic scheme. Therefore, we follow Maia et al. (2016) and continue to refer to these forms as Hepatozoon, pending a complete taxonomic revision of the group.

Similarly controversial is the identification of Hepatozoon parasites in bats. Pinto et al. (2013) provided the first evidence of Hepatozoon parasites infecting Hipposideros cervinus (Gould) from Borneo, with molecular phylogenetic assessments indicating they formed a clade with parasites from reptiles, rodents and marsupials. However, Karadjian et al. (2015) suggest this result should be interpreted with caution. We have observed on several occasions cysts from a haemogregarine in sections of the liver from a species of Miniopterus Bonaparte and considered them to be cysts from a Hepatozoon developing in a dipteran ingested by the bat. Haemogregarine gametocytes were never found in the blood of any bat. These cysts are probably a dead end.

Here we report only the second evidence for species of Hepatozoon from a bat, with a sample from the Zulu pipistrelle being part of a clade with parasites primarily from reptiles and rodents. Screening molecular tissue samples allows far more samples to be included in large-scale assessments than would be available for microscopic ap- proaches. Clearly, the finding of a species of Hepatozoon in two different continents and from two different species means that the original finding of Pinto et al. (2013) cannot be disregarded. Rather, it highlights the need for an extensive, targeted microscopic approach to confirm, whether some species of Hepatozoon can use bats as their vertebrate hosts.

The finding of two very different lineages of Hepatozoon in two species of cobras, Naja mossambica and Naja annulifera, is less unexpected. Various studies have shown that isolated of Hepatozoon identified in snakes reflected in many cases dietary preferences (e.g. Tomé et al. 2014). Similarly, all Hepatozoon from rodents seem to be part of the same clade. Although four different haplotypes were detected from at least four different host species in the present study, they all form part of the same clade, along with all other published sequences retrieved from rodent and many snake hosts.

The detection of isolates of Sarcocystis Lankester, 1882 in tissue samples was also not unexpected, since they have been detected previously in reptile muscle tissue samples using these primers (Harris et al. 2012). One sequence retrieved from an impala was almost identical to sequences from other bovids such as Sarcocystis truncata from red deer, Cervus elaphus. Several studies have proposed that the phylogeny of Sarcocystis reflects the relationships of the final hosts (e.g. Doležel et al. 1999). However, Tomé et al. (2013) have already shown that Sarcocystis from snakes form multiple, unrelated groups. This is important as some authors have attempted to predict definitive hosts based on phylogenetic analysis of the parasite. For example, Tian et al. (2012) proposed a snake as the definitive host of Sarcocystis nesbitti Mandour, 2007 based on their estimate of phylogenetic relationships in which this taxon was sister taxon to Sarcocystis atheridis which does use a snake as the definitive host.

The identification of two distinct lineages from two individuals of white-tailed mongoose shows how complex the situation is and how difficult it is to identify coevolutionary patterns between these parasites and their vertebrate hosts. In our estimate of relationships one parasite lineage from the mongoose is more closely related to $S$. atheridis, whereas the relationship of $S$. nesbitti is poorly supported. Relationships of the Sarcocystis detected in the second white-tailed mongoose, while clearly distinct from the first lineage from this species and from other published sequences from other Sarcocystis, was similarly poorly supported. Sequencing of additional genes for these parasites may help to resolve relationships and confirm if the phylogeny of some of these parasites does reflect that of the final host, or if this only occurs in some groups, such as the bovids.

The remaining parasites detected in the present study were piroplasmids belonging to the families Babesiidae and Theileridae. Although some species of these parasites can infect humans, causing malaria-like symptoms, current knowledge regarding their taxonomy is surprisingly confused. O'Donoghue (2017) proposed that molecular phylogenetic assessments of $18 \mathrm{~S}$ rRNA sequences clear- 
ly distinguished between theileriid and babesiid piroplasmids. Within these, six broad clades were identified, of which theileriid parasites could be split into three, Theileria/Cytauxzoon from felids, Theileria from equids and rhinoceroses, and Theileria from bovids. In our estimate of relationships, separation between theileriid and babesiid piroplasmids is less clear. One possible explanation is that forms were misidentified on GenBank, since errors of this type are not uncommon (Harris 2003). Tissue samples can also be infected with multiple parasites, leading to discrepancies between observed parasites and sequences obtained from infected tissues. Despite these uncertainties, the four samples positive for these parasites in our analysis can be placed within the overall phylogeny with little ambiguity. One sample, from the white-tailed mongoose, was apparently infected with a species of Cytauxzoon, more closely related to Cytauxzoon felis which is widespread in various felids, than to Cytauxzoon manul Reichard, Van Den Bussche, Meinkoth, Hoover et Kocan, 2005 from the Pallas cat, Otocolobus manul (Pallas). Species of Cytauxzoon are common in many felids and rarely reported from other hosts. A similar relationship to the one identified here was reported for a parasite from the Burmese ferret badger Melogale personata Geoffroy Saint-Hilaire, but the sequence is not available for comparisons (Sukmak et al., unpublished conference report). South-African meerkats were also often identified as infected by species of Cytauxzoon, although these were suggested to be the sister taxon to the C.felis/C.manul clade (Leclaire et al. 2015). Further assessments are clearly needed to identify the range of hosts of Cytauxzoon outside of felids, and the relationships between the different species.

The identification of isolate of Babesia Starcovivi, 1893 in the vervet monkey was not unexpected, since this has been previously reported for many African primates (reviewed by Cormier and Jolly 2017). The haplotype was identical to one recovered from an olive baboon, Papio anubis. In contrast, the relationships of the parasites recovered from the ground squirrel Xerus inauris were unexpected. Previously, Babesia microti-like parasites were found in squirrels (e.g. Tsuji et al. 2006). Indeed, although rodents can be infected with very different lineages of Babesia, they are not known to be hosts to species of Theileria (reviewed in O'Donoghue 2017). However, the lineage identified in $X$. inauris is clearly related to Theileria orientalis (Yakimoff and Soudatschenkoff 1931) and other species of Theileria known to infect bovids. The sample from the grass mouse, Rhabdomys sp., is more difficult to place within a phylogenetic framework, because relationships of this new sequence with others are less well supported (Fig. 2).

One sample of white-tailed mongoose was infected with both Hepatozoon and Sarcocystis spp., detected in liver and muscle tissue samples, respectively. Parasite coinfection and interactions can have important influences on the host (e.g. Cattadori et al. 2008). This finding highlights both the value of examining different tissue sources from single animal carcass and also the need for further assessments of apicomplexan parasite interactions within hosts.

Even though conclusive taxonomic inferences regarding the parasites infecting the wide range of hosts included in the present study were not provided, it is clear that, molecular screening repeatedly identifies new lineages of parasites in new hosts, which have profound implications. This highlights the great value of this kind of approach, even if it then requires a follow up with additional markers and, when possible, microscopy to allow more integrative conclusions. As a first step, the method clearly can demonstrate how limited current knowledge still is regarding these important parasites, and can direct further studies to key hosts that have been previously overlooked.

Acknowledgements. DJH and RX are supported by the Fundação para a Ciência e a Tecnologia (FCT, Portugal) through IF contracts $01627 / 2014$ and $00359 / 2015$, respectively. Thanks are due to Chris Burger for identification of snakes, Philippe Gaubert and Emmanuel Do Linh San for confirming the identification of genets, and to all those who helped $\mathrm{AH}$ with roadkills and checking them: Wilmien J Luus-Powell, David Kunutu, Michael Rampedi, Makubu P. Mokgawa, Sello Matjee (University of Limpopo) and Jabu Linden (Lajuma Research Centre).

\section{REFERENCES}

Basson P.A., McCully R.M., Bigalke R.D., van Niekerk J.W. 1967: Observations on a Hepatozoon-like parasite in the impala. J. S. Afr. Vet. Assoc. 38: 12-13.

Bosman A.M., Oosthuizen M.C., Peirce M.A., Venter E.H., Penzhorn B.L. 2010: Babesia lengau sp. nov., a novel Babesia species in cheetah (Acinonyx jubatus Schreber, 1775) populations in South Africa. J. Clin. Microbiol. 48: 2703-2708.

Cattadori I.M., Boag B., Hudson P.J. 2008: Parasite co-infection and interaction as drivers of host heterogeneity. Int. J. Parasitol. 38: 371-380.

Cook C.A., Netherlands E.C., Smit N.J. 2016: Redescription, molecular characterisation and taxonomic re-evaluation of a unique African monitor lizard haemogregarine Karyolysus paradoxa (Dias, 1954) n. comb. (Karyolysidae). Parasit. Vectors 9: 347.

Cormier L.A., Jolly P.E. 2017: The Primate Zoonoses: Culture Change and Emerging Diseases. Routledge, New York, 144 pp.
Criscione C., Poulin R., Blouin M. 2005: Molecular ecology of parasites: elucidating ecological and microevolutionary processes. Mol. Ecol. 14: 2247-2257.

Dobson A., Lafferty K.D., Kuris A.M., Hechinger R.F., JETZ W. 2008: Homage to Linnaeus: how many parasites? How many hosts? Proc. Natl. Acad. Sci. U.S.A. 105: 11482-11489.

Doležel D., Koudela B., JirkŮ M., Hypša V., Oborník M., Votýpka J., ModrÝ D., ŠLapeta J.T., LukeŠ J. 1999: Phylogenetic analysis of Sarcocystis spp. of mammals and reptiles supports the coevolution of Sarcocystis spp. with their final hosts. Int. J. Parasitol. 29: 795-798.

Dubey J.P., Lane E.P., van Wilpe E., Suleman E., Reininghaus B., Verma S.K., Rosenthal B.M., Mtshali M.S. 2014: Sarcocystis cafferi n. sp. (Protozoa: Apicomplexa) from the African buffalo (Syncerus caffer). J. Parasitol. 100: 817-827 
Dunn R.R., Har Ris N.C., Colwell R.K., Koh L.P., Sodhi N.S. 2009: The sixth mass coextinction: are most endangered species parasites and mutualists? Proc. Biol. Sci. 276: 3037-45.

HaRris D.J. 2003: Can you bank on GenBank? Trends Ecol. Evol. 18: 317-319.

Harris D.J., Graciá E., Jorge F., Maia J.P.M.C., Perera A., Carretero M.A., Giménez A. 2013: Molecular detection of Hemolivia (Apicomplexa: Haemogregarinidae) from ticks of North African Testudo graeca (Testudines: Testudinidae) and an estimation of their phylogenetic relationships using 18S rRNA sequences. Comp. Parasitol. 80: 292-296.

Harris D.J., Maia J.P.M.C., Perera A. 2012: Molecular survey of Apicomplexa in Podarcis wall lizards detects Hepatozoon, Sarcocystis, and Eimeria species. J Parasitol. 98: 592-597.

Harris D.J., Pereira A., Halajian A., Luus-Powell W.J., Kunutu K.D. 2017: Screening for Hepatozoon parasites in gerbils and potential predators in South Africa. J. S. Afr. Vet. Assoc. 88: $1-4$.

Hoverman J.T., Mihaljevic J.R., Richgels K.L.D., Kerby J.L., Johnson P.T.J. 2012: Widespread co-occurrence of virulent pathogens within California amphibian communities. Ecohealth 9: 288-292.

Huelsenbeck J.P., Ronquist F. 2001: Mr. Bayes: Bayesian inference of phylogeny. Bioinformatics 17: 754-755.

Karadjian G., Chavatte J.-M., Landau I. 2015: Systematic revision of the adeleid haemogregarines, with creation of Bartazoon n. g., reassignment of Hepatozoon argantis Garnham, 1954 to Hemolivia, and molecular data on Hemolivia stellata. Parasite 22: $1-15$.

Lanfear R., Calcott B., Ho S.Y.W., Guindon S. 2012: PartitionFinder: combined selection of partitioning schemes and substitution models for phylogenetic analyses. Mol. Biol. Evol. 29: $1695-1701$.

Leclaire S., Menard S., Berry A. 2015: Molecular characterisation of Babesia and Cytauxzoon species in wild South-African meerkats. Parasitology 142: 543-548.

Maia J.P., Carranza S., Harris D.J. 2016: Comments on the systematic revision of adeleid haemogregarines: is more data needed? J. Parasitol. 102: 549-552.

Maia J.P., Harris D.J., Carranza S., Gómez-Díaz E. 2014: A comparison of multiple methods for estimating parasitemia of hemogregarine hemoparasites (Apicomplexa: Adeleorina) and its application for studying infection in natural populations. PLoS ONE 9: e95010.

Maia J.P.M.C., Harris D.J., Perera A. 2011: Molecular survey of Hepatozoon specie in lizards from North Africa. J. Parasitol. 97: 513-517.

Mittermeier R.A., Gils P.R., Hoffman M., Pilgrim J., Brooks T., Mittermeier C.G., Lamoreux J., da Fonseca G.A.B. 2004: Hotspots Revisited: Earth's Biologically Richest and Most Endangered Terrestrial Ecoregions. CEMEX, Mexico City, $392 \mathrm{pp}$.

Netherlands E.C. Cook C.A., Smit N.J. 2014: Hepatozoon species (Adeleorina: Hepatozoidae) of African bufonids, with morphological description and molecular diagnosis of Hepatozoon ixoxo sp. nov. parasitising three Amietophrynus species (Anura: Bufonidae). Parasit. Vectors 7: 552.

O'Donoghue P. 2017: Haemoprotozoa: making biological sense of molecular phylogenies. Int. J. Parasitol. Parasites Wildl. 6: 241256.

Pedersen A.B., Fenton A. 2007: Emphasising the ecology in parasite community ecology. Trends Ecol. Evol. 22: 133-139.

Pinto C.M., Helgen K.M., Fleischer R.C., Perkins S.L. 2013: Hepatozoon parasites (Apicomplexa: Adeleorina) in bats. J. Parasitol. 99: 722-724.

Sloboda M., Kamler M,. Bulantová J., VotÝPKa J., ModrÝ D. 2007: A new species of Hepatozoon (Apicomplexa: Adeleorina) from Python regius (Serpentes: Pythonidae) and its experimental transmission by mosquito vector. J. Parasitol. 93: 1189-1198.

Smith T.G., Desser S.S. 1997: Phylogenetic analysis of the genus Hepatozoon Miller, 1908 (Apicomplexa: Adeleorina). Syst. Parasitol. 36: 213-221.

Stamatakis A. 2006: RAxML-VI-HPC: maximum likelihood-based phylogenetic analyses with thousands of taxa and mixed models. Bioinformatics 22: 2688-90.

Telford S. R. 2009: Hemoparasites of the Reptilia: Color Atlas and Text. CRC Press, Boca Raton, Florida, 376 pp.

Tian M., Chen Y., Wu L., Rosenthal B.M., Liu X., He Y., Dunams D.B., Cui L., Yang Z. 2012: Phylogenetic analysis of Sarcocystis nesbitti (Coccidia: Sarcocystidae) suggests a snake as its probable definitive host. Vet. Parasitol. 183: 373-376.

Tomé B., Maia J.P., Salvi D., Brito J.C., Carretero M.A, Perera A., Meimberg H., Harris D.J. 2014: Patterns of genetic diversity in Hepatozoon spp. infecting snakes from North Africa and the Mediterranean Basin. Syst. Parasitol. 87: 249-58.

Tomé B., Rato C., Perera A., Harris D.J. 2016: High diversity of Hepatozoon spp. in geckos of the genus Tarentola. J. Parasitol. 102: 476-480.

Tsuji M., Zamoto A., Kawabuchi T., Kataoka T., NaKajima R., Asakawa M., Ishinara C. 2006: Babesia microti-like parasites detected in Eurasian red squirrels (Sciurus vulgaris orientis) in Hokkaido, Japan. J. Vet. Med. Sci. 68: 643-646.

Ujvari B., Madsen T., Olsson M. 2004: High prevalence of Hepatozoon spp. (Apicomplexa, Hepatozoidae) infection in water pythons (Liasis fuscus) from tropical Australia. J. Parasitol. 90: 670-672.

Van As J., Davies A.J., Smit N.J. 2013: Hepatozoon langii n. sp. and Hepatozoon vacuolatus n. sp. (Apicomplexa: Adeleorina: Hepatozoidae) from the crag lizard (Sauria: Cordylidae) Pseudocordylus langi from the North Eastern Drakensberg escarpment, Eastern Free State, South Africa. Zootaxa 3608: 345-356.

Cite this article as: Harris D.J., Halajian A., Santos J.L., Swanepoel L.H., Taylor P.J., Xavier R. 2018: Diversity of haemoprotozoan parasites infecting the wildlife of South Africa. Folia Parasitol. 65: 015 